Logtenberg, S.L.M., Vink, M.L., Godfried, M.B., Beenakkers, I.C.M., Schellevis, F.G., Mol, B.W., Verhoeven, C.J. Serious adverse events attributed to remifentanil patient-controlled analgesia during labour in The Netherlands. International Journal of obstetric Anesthesia: 2019, 3, p. 22-28

$\begin{array}{lll}\text { Postprint version } & : \\ \text { Journal website } & : \frac{\text { https://www.obstetanesthesia.com/article/S0959-289X(18)30216- }}{4 / \text { fulltext }} \\ \text { Pubmed link } & : \frac{\text { https://www.ncbi.nlm.nih.gov/pubmed/30509681 }}{\text { DOI }} & : 10.1016 / \text { j.ijoa.2018.10.013 }\end{array}$

This is a Nivel certified Post Print, more info at nivel.nl

\title{
Serious adverse events attributed to remifentanil patient-controlled analgesia during labour in The Netherlands
}

\author{
S.L.M.Logtenberg, ${ }^{\text {ab }}$ M.L.Vink, ${ }^{\text {C M.B.Godfried, }}{ }^{\text {I I.C.M.Beenakkers, }}{ }^{e}$ \\ F.G.Schellevis, ${ }^{\mathrm{fg}}{ }^{\text {B.W.Mol, }}{ }^{\mathrm{h}}$ C.J.Verhoeven, ${ }^{\mathrm{ij}}$
}

${ }^{a}$ Amsterdam UMC, Academic Medical Centre, Department of Obstetrics and Gynaecology, Amsterdam, The Netherlands

${ }^{\mathrm{b}}$ AVAG Amsterdam, Amsterdam, The Netherlands

'Midwifery Practice Haarlemmermeer \& Bollenstreek, Nieuw Vennep, The Netherlands dDepartment of Anesthesiology, OLVG, Amsterdam, The Netherlands

eDepartment of Anesthesiology, Wilhelmina Children's Hospital, Utrecht, The Netherlands ${ }^{\dagger}$ Amsterdam UMC, Vrije Universiteit Amsterdam, Department of General Practice \& Elderly Care Medicine, Amsterdam Public Health Research Institute, Amsterdam, The Netherlands ${ }^{9}$ Netherlands Institute for Health Services Research (NIVEL), Utrecht, The Netherlands hepartment of Obstetrics and Gynaecology, Monash University Clayton, Clayton, Victoria, Australia

'Amsterdam UMC, Vrije Universiteit Amsterdam, Department of Midwifery Science, AVAG, Amsterdam Public Health Research Institute, Amsterdam, The Netherlands 'Maxima Medical Centre, Veldhoven, The Netherlands

\footnotetext{
Abstract

Background:

During labour, remifentanil patient-controlled analgesia is used as an alternative to neuraxial analgesia. Remifentanil is associated with hypoventilation and respiratory depression but the frequency of serious maternal and neonatal adverse events is unknown. The aim of this study was to estimate the number of serious adverse events attributed to the use of remifentanil patient-controlled analgesia during labour in The Netherlands and to investigate the circumstances (e.g. monitoring, practice deviations) of these events and the subsequent management.

Methods:

In a nationwide survey among obstetricians, anaesthetists and clinical midwives the frequency of serious adverse events was assessed. A questionnaire was sent by email to
} 
Logtenberg, S.L.M., Vink, M.L., Godfried, M.B., Beenakkers, I.C.M., Schellevis, F.G., Mol, B.W., Verhoeven, C.J. Serious adverse events attributed to remifentanil patient-controlled analgesia during labour in The Netherlands. International Journal of obstetric Anesthesia: 2019, 3, p. 22-28

all 61 Dutch hospitals in which remifentanil patient-controlled analgesia is, or has been, available for labour analgesia. All reported cases were assessed independently by two expert teams.

Results:

We received information from all hospitals. After independent assessments, 17 cases of single maternal desaturation; 10 maternal cases of apnoea, bradycardia and/or cardiac arrest; and two neonatal cases of respiratory depression, over a period of more than 10 years of remifentanil patient-controlled analgesia use, were identified as a serious adverse event. All serious adverse events were resolved without irreversible damage. Conclusions:

The risk of a potentially life-threatening serious adverse event attributed to remifentanil patient-controlled analgesia seems to be low. All patients recovered without deficit. Adherence to strict monitoring and the attendance of trained healthcare providers is required to safely use remifentanil for labour analgesia.

\section{Introduction}

Epidural analgesia is considered to be the most effective and a preferred method of labour analgesia.1, 2 Remifentanil, a synthetic opioid, has a fast onset of action, short half-life and is metabolised and redistributed quickly by the fetus.3, 4 These properties make remifentanil patient-controlled analgesia (PCA) an alternative to epidural analgesia when not available, not desired or contraindicated, although its use is considered off-label. ${ }^{5}$ Remifentanil PCA has been used for labour analgesia over the past decade in Europe.6, 7, 8, 9, 10, 11 However, remifentanil PCA has been associated with hypoventilation and respiratory depression. ${ }^{3}$ Several case reports have been published in which maternal respiratory arrest, and/or a cardiac arrest, was attributed to the use of remifentanil PCA.12, $13,14,15,16,17$ Moreover, Kan et al. found that remifentanil crosses the placenta rapidly and may theoretically cause neonatal respiratory depression. ${ }^{3}$ A recent Cochrane review recommended further research on the maternal and neonatal safety of remifentanil PCA during labour. ${ }^{18}$

Due to these safety concerns, remifentanil PCA is considered to be a controversial method of labour analgesia.19, 20 In the Dutch Societies of Obstetrics and Gynaecology and Anaesthesiology guideline for labour analgesia, the risk of incautious use of remifentanil PCA is mentioned.2, 11 Safety concerns and the variety of remifentanil PCA administration protocols for labour analgesia led to mandatory implementation of a multidisciplinary Standard Operating Procedure (SOP) by the Dutch Health Care Inspectorate, in every Dutch hospital. The SOP was composed and introduced by the Dutch Societies of Obstetrics and Gynaecology, Midwifery, Anaesthesiology and Hospital Pharmacists in $2014 .{ }^{21}$ Since introduction of this SOP, no evaluation has been performed to assess the use of remifentanil PCA during labour.

The frequency of serious adverse events (SAEs) such as maternal apnoea, bradycardia and cardiac arrest, as well as neonatal respiratory depression, bradycardia and cardiac arrest as a result of remifentanil PCA during labour, is unknown. More knowledge about the frequency of maternal and neonatal SAEs attributed to the use of remifentanil PCA during labour and the circumstances of these cases is needed to validate this as a suitable method of labour analgesia and for the counselling of pregnant women.

The aims of this study were to estimate the number of serious maternal and neonatal adverse events attributed to the use of remifentanil PCA during labour; and to investigate the clinical circumstances (e.g. monitoring, deviations from the SOP) of these cases and the procedures followed in managing the events. 
Logtenberg, S.L.M., Vink, M.L., Godfried, M.B., Beenakkers, I.C.M., Schellevis, F.G., Mol, B.W., Verhoeven, C.J. Serious adverse events attributed to remifentanil patient-controlled analgesia during labour in The Netherlands. International Journal of obstetric Anesthesia: 2019, 3, p. 22-28

\section{Methods}

We conducted a descriptive study. Information about possible SAEs attributed to the use of remifentanil PCA was collected through an online questionnaire. The circumstances of the possible SAEs, and the procedures followed after the events, were assessed independently by two expert teams, each of which consisted of an obstetrician, an anaesthetist and a clinical midwife. Our study did not require formal approval of an ethics committee, according to Dutch law, as confirmed by the ethics committee of the Academic Medical Centre in Amsterdam (ref.nr. W17_427\#17.495). We developed the online questionnaire in LimeSurvey. ${ }^{22}$ Links to the questionnaire were sent by email to healthcare providers involved in the administration of remifentanil PCA, namely one obstetrician, one anaesthetist and one clinical midwife in each of the 61 Dutch hospitals where remifentanil PCA is or has been available for labour analgesia. We have previously reported, in a survey about remifentanil PCA practices sent to obstetricians in all 81 Dutch hospitals with a labour ward, that 59 (73\%) have remifentanil PCA available, and that in two hospitals it had previously been available. ${ }^{9}$ Of these 59 hospitals, six are academic, 26 non-academic teaching and 27 are non-teaching hospitals. One academic hospital used remifentanil PCA only during the RAVEL trials between 2011 and 2013 and one non-academic teaching hospital discontinued remifentanil PCA in 2012. ${ }^{9}$ Before sending the questionnaire we contacted all hospitals to enquire about the most suitable obstetrician, anaesthetist and clinical midwife in each institution to answer the questionnaire. After the first invitation, two reminder emails were sent to each potential respondent, each after two weeks. In case of no response after these reminders, we made a telephone approach.

The questionnaire included two multiple-choice questions with the option of free text. To ensure validity and comprehensiveness, we piloted the questionnaire among four obstetricians, four anaesthetists and four clinical midwives from both academic and non-academic hospitals. This pilot led to some linguistic revisions.

After revisions the questions were: "To the best of your knowledge, have there been any incidents and/or complications at your institution of maternal respiratory depression or respiratory arrest and/or bradycardia or cardiac arrest, possibly as a result of the use of remifentanil PCA during labour (since the start of the use of remifentanil PCA for labour analgesia in your department)?" and "To the best of your knowledge, have there been any incidents and/or complications at your institution of neonatal respiratory depression or respiratory arrest and/or bradycardia or cardiac arrest, possibly as a result of the use of remifentanil PCA during labour (since the start of the use of remifentanil PCA for labour analgesia in your department)?". We adopted a broad description of an SAE, aiming to substantiate all potential cases. If the respondent reported a possible SAE, we requested further details about the situation in which the possible SAE had occurred. Furthermore, if a possible SAE was reported, we asked for the name of the healthcare provider responsible for the case.

We contacted the healthcare provider for additional information, preferably based on the patient record, using a checklist. The checklist contained items such as the calendar year of the SAE, the maternal and neonatal vital signs, rescue treatments, medications and obstetric outcomes. We asked for specific details, based on previous case reports, such as use of supplemental oxygen or a background remifentanil infusion, administration of an opioid less than four hours before the start of remifentanil PCA, one-to-one care and if retrospective checks for pump and medication failure had been conducted.12,13,14,15,16, 17 The respondents could select the option that they were unaware of any SAE or that she/he did not have information about possible SAEs. In the latter situation we requested the contact details of a colleague to verify the answer. Furthermore, the respondent had the option to state that she/he did not want to answer the questions. Details of the women with SAEs were reported anonymously, and we based our information only on written and verbal information from the healthcare providers. References to the identity of the caregiver and the hospital were deleted prior to analyses. If we received more responses from the same hospital we contacted the respondents to verify whether these were duplicated reports of the same cases. 
Logtenberg, S.L.M., Vink, M.L., Godfried, M.B., Beenakkers, I.C.M., Schellevis, F.G., Mol, B.W., Verhoeven, C.J. Serious adverse events attributed to remifentanil patient-controlled analgesia during labour in The Netherlands. International Journal of obstetric Anesthesia: 2019, 3, p. 22-28

The Dutch SOP prescribes requirements for the use of remifentanil PCA, such as education for healthcare providers, the procedure to obtain informed consent, maternal monitoring requirements, preparation for the application of the method, treatment for complications and documentation. Since the literature does not provide an operational definition of an SAE related to remifentanil use, we established our two expert teams. The first expert team had two tasks. Prior to data collection they were asked to define maternal and neonatal respiratory depression and arrest, bradycardia and cardiac arrest, based on the literature and their clinical experience. ${ }^{23}$ Maternal respiratory depression or arrest was defined as an oxygen saturation of $85 \%$ or less $\left(\mathrm{SpO}_{2} \leq 85 \%\right)$ and/or an apnoea lasting at least 20 seconds (respiratory rate $\leq 3 /$ minute) and/or the application of bag-mask ventilation. Maternal bradycardia was defined as a heart rate of 50 beats/min or less and cardiac arrest as the absence of maternal pulse, for which cardiopulmonary resuscitation (CPR) was applied; and neonatal respiratory depression or arrest as apnoea for which bag-mask ventilation was applied. The definition of neonatal bradycardia was a heart rate of 60 beats/min or less and cardiac arrest as the absence of neonatal pulse, for which neonatal cardiopulmonary resuscitation was applied.

Subsequently, both expert teams independently reviewed all possible SAEs and using any additional information provided, assessed whether a reported case was likely an SAE attributed to the use of remifentanil PCA. The expert teams used the written information and the information collected via checklists, and the definitions determined by the first expert team for these assessments.

Discrepancies were resolved by a third anaesthetist who independently judged these cases. In some cases the additional information was insufficient to determine whether it had been an SAE; for this reason, the responses required interpretation. Additionally, a paediatrician was contacted to independently review all neonatal cases. To ascertain complete reporting of these SAEs attributed to remifentanil PCA, we checked two other sources where SAEs could have been registered or reported, these being the Dutch Health Care Inspectorate and Lareb (The Dutch Pharmacovigilance Centre). This information was obtained by personal contact (SL).

\section{Results}

Between January and March 2018 the questionnaire was completed by 61/61 (100\%) obstetricians; $54 / 61(89 \%)$ anaesthetists and by 59/61 (97\%) clinical midwives. We received 36 reports of a possible maternal SAE and four reports of a possible neonatal SAE attributed to the use of remifentanil PCA for labour analgesia. Additional information about the reported cases was retrieved from the patient record for 18 maternal and one neonatal case; and was based on the respondent's memory for 12 maternal and three neonatal cases. For six maternal cases the respondents declined consent to be approached for additional information. Information was mostly provided by the healthcare providers responsible for the case of the possible SAE. Both expert teams assessed all maternal and neonatal reports. Fig. 1 shows the outcome after the assessments by the expert teams.

\section{[figure 1]}

Of the 23 maternal cases involving oxygen desaturation, 17 were single events and were treated by encouraging breathing and/or discontinuation of remifentanil PCA and/or supplemental oxygen. In five cases desaturation occurred in combination with apnoea and in all these five cases a background infusion was used simultaneously with remifentanil PCA boluses. In two cases of apnoea the administration of remifentanil PCA was discontinued and supplemental oxygen was applied. In one case bag-mask ventilation was applied and naloxone given intravenously. This woman had simultaneous administration of other medications: magnesium sulphate; oxytocin; methyldopa and nifedipine. In one case intubation was performed because effective bag-mask ventilation was not possible and one woman with oxygen desaturation and apnoea was treated with three thoracic compressions, without other interventions. One woman had oxygen desaturation, apnoea and a 
Logtenberg, S.L.M., Vink, M.L., Godfried, M.B., Beenakkers, I.C.M., Schellevis, F.G., Mol, B.W., Verhoeven, C.J. Serious adverse events attributed to remifentanil patient-controlled analgesia during labour in The Netherlands. International Journal of obstetric Anesthesia: 2019, 3, p. 22-28

cardiac arrest, and cardiopulmonary resuscitation was applied for three minutes. In this case, in retrospect, $10 \mathrm{~mL}$ of the drug appeared to be missing from the syringe. An overdose of remifentanil might have been caused by a single error of the PCA pump or during the connection of the perfusor line to the patient (Table 1). All women with an SAE recovered completely, without deficit.

\section{[table 1]}

In one neonatal case of apnoea a stiff thorax was diagnosed. This was considered to be the cause of the respiratory depression that necessitated intubation. In the second neonatal case, delivery occurred within three minutes of the last bolus of remifentanil PCA. Five inflation breaths and positive endexpiratory pressure were applied, after which the neonate did not need further resuscitation (Table 1). None of the reported neonatal adverse events occurred in a mother with an adverse event. Both neonates with an SAE recovered completely, with no deficit at the end of the treatment. No cases of maternal or neonatal SAEs had been reported to the Dutch Health Care Inspectorate. Lareb had registered six cases of 'maternal side effects' of remifentanil PCA during labour between 2004 and 2017. This registration consisted of three cases of skin rash; one case of oxygen desaturation; one case of respiratory depression and one case of cardiac arrest. It is not known if these were among the cases reported by the healthcare providers, or if they were additional cases. Additional information about these cases was not available.

\section{Discussion}

We studied the number of SAEs attributed to the use of remifentanil PCA during labour in The Netherlands. In our survey among obstetricians, anaesthetists and clinical midwives we identified 27 maternal and two neonatal SAE cases. The 27 maternal cases comprised 23 desaturation events, six apnoea events, four bradycardia events and one cardiac arrest. The two neonatal cases both concerned respiratory depression. In five cases of maternal apnoea, a background infusion was running in addition to remifentanil PCA boluses. All SAEs were managed without lasting harm during the hospital stay.

Our study provides an opportunity to estimate the frequency of SAEs attributed to remifentanil PCA in The Netherlands. Although our observation of SAE frequency is likely to be an underestimation, the risk of an SAE attributed to remifentanil PCA seems to be low. Aaronson et al. found 14 complications in 340 cases of remifentanil PCA use during one year. ${ }^{17}$ In comparison, 21000 women per year received remifentanil PCA in The Netherlands in 2016 and in $2017 .{ }^{\circ}$ The frequency of SAEs in this study corresponds to that reported by Melber et al. ${ }^{24}$ Nevertheless, despite the introduction of an SOP in The Netherlands, SAEs associated with the use of remifentanil PCA for labour analgesia still occur. Serious adverse events are acute and severe and necessitate immediate treatment. Strict monitoring during remifentanil PCA and the attendance of trained healthcare providers is required to identify and manage SAEs.21, 23, 25 Optimal maternal monitoring regimens during remifentanil labour analgesia remain to be determined.19, 26 Weiniger et al. found that only $15 \%$ of apnoea events were detected by the threshold trigger of $<92 \%$, using a pulse oximetry device. ${ }^{23}$ This could explain the underestimation of desaturation events. In the Netherlands it is common clinical practice to detect apnoea by measuring oxygen saturation with pulse oximetry during remifentanil PCA use. Most apnoea events reported by Weiniger et al. during remifentanil PCA use were detected by capnography or by the Integrated Pulmonary Index (a combination score from respiratory and heart rates, oxygen saturation and end-tidal carbon dioxide). ${ }^{23}$ Messmer et al. found a $10 \%$ incidence of extreme oxygen desaturation ( $<80 \%$ ) in women using remifentanil PCA and a $70 \%$ incidence of desaturation to less than $90 \%$. In all those cases, the woman recovered spontaneously. ${ }^{27}$ The 17 cases of a single episode of desaturation below $85 \%$ in our study are likely to be an underestimation. It is unknown as to what extent one-to-one care is used throughout remifentanil PCA administration in The Netherlands. 
Logtenberg, S.L.M., Vink, M.L., Godfried, M.B., Beenakkers, I.C.M., Schellevis, F.G., Mol, B.W., Verhoeven, C.J. Serious adverse events attributed to remifentanil patient-controlled analgesia during labour in The Netherlands. International Journal of obstetric Anesthesia: 2019, 3, p. 22-28

Although, according to the Dutch SOP, one-to-one care is not mandatory after the first hour of remifentanil administration, it is likely that continuous one-to-one care prevents apnoea. A balance between strict maternal monitoring and feasibility for healthcare providers is needed when remifentanil is used for labour analgesia. If maternal monitoring cannot be fully accomplished, remifentanil PCA should not be administered.

In five cases of apnoea (that occurred prior to the introduction of the SOP) a background infusion of remifentanil was used in addition to a bolus demand dose. A background infusion is disallowed in the SOP, so it is surprising that some Dutch hospitals are still using a background infusion. ${ }^{9}$ In previously reported cases with a SAE, a background infusion was also used.13, 15, 17 Some of our reported cases included human or technical errors, such as failure of the alarm within the monitoring system; an incorrectly adjusted infusion pump; and an infusion pump error. These probable causes of an SAE are comparable with the studies of Kinney et al. and Aaronson et al., where medication errors were reported.13, 17 Despite safeguards such as the national SOP, such a safeguard does not prevent some potentially life-threatening errors that can be detected by alert healthcare providers. Since the introduction of the SOP, one maternal case of oxygen desaturation, one maternal case of apnoea, three maternal cases of bradycardia and two neonatal SAEs have been described. The woman with apnoea received magnesium sulphate as well as remifentanil, increasing the risk of respiratory depression. According to the SOP, magnesium sulphate is a relative contraindication for the use of remifentanil PCA. Bradycardia may occur, even when SOP guidelines are followed. In addition, systematic registration of the use of remifentanil PCA, as well as of SAEs, is required in order to be able to evaluate the safety of remifentanil PCA.

This is the first study to have investigated SAEs attributable to remifentanil PCA during labour in The Netherlands. The main strength of the study is the excellent response rate. We received information from all hospitals where remifentanil PCA was or is used for labour analgesia, and also reports of SAEs from the Dutch Health Care Inspectorate and Lareb. Although screening of a patient's medical records would have provided a more complete picture of SAEs, this would have been time-consuming and expensive, given the number of times remifentanil PCA was used. Furthermore, all cases were assessed by two independent expert teams and the neonatal cases by a paediatrician, which contributed to the internal validity of the study.

Our study also has weaknesses, in particular that the number of reported SAEs is likely to be an underestimate. A reason for this could be that maternal monitoring, according to the SOP, is implemented by only $28(48 \%)$ of the 59 Dutch hospitals in which remifentanil PCA is available.9, 28 In addition, due to the definition of oxygen desaturation used ( $\mathrm{an} \mathrm{SpO}_{2} \leq 85 \%$ ), oxygen saturation values between $85 \%$ and $94 \%$ were not included as events. Finally, response bias could have occurred, as some respondents may have been unaware of SAEs or reluctant to report them. Most of the reported cases date from several years ago and in several, information was provided without confirmation using the patient's medical record or was too limited to be assessed.

In conclusion, the number of reported potentially life-threatening SAEs attributed to remifentanil PCA as labour analgesia was low. The adherence to strict maternal monitoring and the attendance of trained healthcare providers are an essential requirement for the safe use of remifentanil PCA during labour.

\section{Funding}

This research did not receive any specific grant from funding agencies in the public, commercial, or not-for-profit sectors.

Recommended articlesCiting articles (0) 
Logtenberg, S.L.M., Vink, M.L., Godfried, M.B., Beenakkers, I.C.M., Schellevis, F.G., Mol, B.W., Verhoeven, C.J. Serious adverse events attributed to remifentanil patient-controlled analgesia during labour in The Netherlands. International Journal of obstetric Anesthesia: 2019, 3, p. 22-28

\section{References}

1. ACOG. Practice Bulletin No. 177: Obstetric Analgesia and Anesthesia. Obstet Gynecol 2017;129:e73-89.

2. NVOG Dutch Society of Obstetrics and Gynaecology. Guideline pain relief during labour (pijnstilling tijdens de bevalling). 2008.

3. Kan RE, Hughes SC, Rosen MA, Kessin C, Preston PG, Lobo EP. Intravenous remifentanil: placental transfer, maternal and neonatal effects. Anesthesiology 1998;88:1467-74.

4. Egan TD. Pharmacokinetics and pharmacodynamics of remifentanil: an update in the year 2000. Curr Opin Anaesthesiol 2000;13:449-55.

5. CBG. Public Assessment Report Remifentanil (2010).

6. Saravanakumar K, Garstang JS, Hasan K. Intravenous patientcontrolled analgesia for labour: a survey of UK practice. Int J Obstet Anesth 2007;16:221-5.

7. Tveit TO, Halvorsen A, Rosland JH. Analgesia for labour: a survey of Norwegian practice -With a focus on parenteral opioids. Acta Anaesthesiol Scand 2009;53:494-9.

8. Schnabel A, Hahn N, Muellenbach R, et al. Obstetric analgesia in German clinics. Remifentanil as alternative to regional analgesia. Der Anaesthesist 2011;60:995-1001.

9. Logtenberg S, Vink L, Godfried M, et al. Practice variation in remifentanil during labour; and overview of its application in Dutch hospitals. Ned Tijdschr Geneeskd 2018;162. pii: D2816.

10. Freeman LM, Dahan A, van Lith JMM, et al. Epidural analgesia versus remifentanil patient controlled analgesia in labor: a survey of practice in the Netherlands. Int $\mathrm{J}$ Clin Anesthesiol 2016;4:1051.

11. Inspectie voor de Gezondheidszorg. Thematisch toezicht geboortezorg: Afsluitend onderzoek naar de invoering van de normen van 'Een goed begin'. (2016). Available at:

https://www.igj.nl/documenten/rapporten/2016/04/19/thematisch-toezicht-geboortezorgafsluitendonderzoek-naar-de-invoering-van-de-normen-van-'eengoed-begin'. Accessed 5th October 2017.

12. Bonner JC, McClymont W. Respiratory arrest in an obstetric patient using remifentanil patientcontrolled analgesia. Anaesthesia 2012;67:538-40.

13. O'Kinney MA, Rose CH, Traynor KD, et al. Emergency bedside cesarean delivery: lessons learned in teamwork and patient safety. BMC Research Notes 2012;5:412-6.

14. Marr R, Hyams J, Bythell V. Cardiac arrest in an obstetric patient using remifentanil patientcontrolled analgesia. Anaesthesia 2013;68:283-7.

15. Waring J, Mahboobi SK, Tyagaraj K, Edd D. Use of remifentanil for labor analgesia: the good and the bad. Anesth Analg 2007;104:1616-7.

16. Pruefer C, Bewlay A. Respiratory arrest with remifentanil patientcontrolled analgesia - another case. Anaesthesia 2012;67:1044-5.

17. Aaronson J, Abramovitz S, Smiley R, Tangel V, Landau R. A survey of intravenous remifentanil use for labor analgesia at academic medical centers in the United States. Anesth Analg 2017;124:1208-10.

18. Weibel $S$, Jelting $Y$, Afshari $A$, et al. Patient-controlled analgesia with remifentanil versus alternative parenteral methods for pain management in labour. Cochrane Database Syst Rev 2017;4: CD011989.

19. Van De Velde M. Remifentanil Patient-controlled intravenous analgesia for labor pain relief: is It really an option to consider? Anesth Analg 2017;124:1029-31.

20. Van De Velde M. Patient-controlled intravenous analgesia remifentanil fot labor analgesia: time to stop, think and reconsider. Curr Opin Anaesthesiol 2015;28:237-9.

21. NVOG Dutch Society of Obstetrics and Gynaecology, NVA Dutch Society of Anaesthesiology, KNOV Dutch Society of Midwifery \& NVZA Dutch Society of Hospital Pharmacists. Standard

Operation Procedure Remifentanil PCA. (2014)

22. Lime Survey. Available at: https://www.limesurvey.org/. Accessed Oct 2018. 
Logtenberg, S.L.M., Vink, M.L., Godfried, M.B., Beenakkers, I.C.M., Schellevis, F.G., Mol, B.W., Verhoeven, C.J. Serious adverse events attributed to remifentanil patient-controlled analgesia during labour in The Netherlands. International Journal of obstetric Anesthesia: 2019, 3, p. 22-28

23. Weiniger CF, Carvalho B, Stocki D, Einav S. Analysis of physiological respiratory variable alarm alerts among labouring women receiving remifentanil. Anesth Analg 2017;124:1211-8. 24. Melber AA, Sia ATH. "Do no harm" - Where to place remifentanil for labour analgesia? Trends Anaesth Crit Care 2017;17:17-20.

25. Van De Velde M, Carvalho B. Remifentanil for labor analgesia: an evidence-based narrative review. Int J Obstet Anesth 2016;25:66-74.

26. Beenakkers ICM, Douma MR, Kam-Endz CE, Freeman LM. Remifentanil during labour; has its place in pain relief during labour been decided? Ned Tijdschr Geneeskd 2018;162. pii:D1948. 27. Messmer AA, Potts JM, Orlikowski CE. A prospective observational study of maternal oxygenation during remifentanil patientcontrolled analgesia use in labour. Anaesthesia 2016;71:171-6.

28. Hoenen EEJN, Wassen MMLH, Nijhuis JG, Rijke RPC. Naleving van de 'Standard Operating Procedure Remifentanil Patient controlled Analgesia' in Nederland. Dutch J Obstet Gynaecol 2018;131:294-8.

\section{Figures and tables}

Fig. 1. Flowchart of cases with one or more maternal or neonatal serious adverse events. *One case could contain more than one serious adverse event. Desaturation $=$ oxygen saturation $\leq 85 \%$; maternal apnoea = respiratory rate $\leq 3 /$ minute; neonatal apnoea = need for bag-mask ventilation; maternal bradycardia = heart rate $\leq 50$ beats/minute; cardiac arrest = absence of maternal pulse. SAE: serious adverse event; remifentanil PCA: remifentanil patient-controlled intravenous analgesia

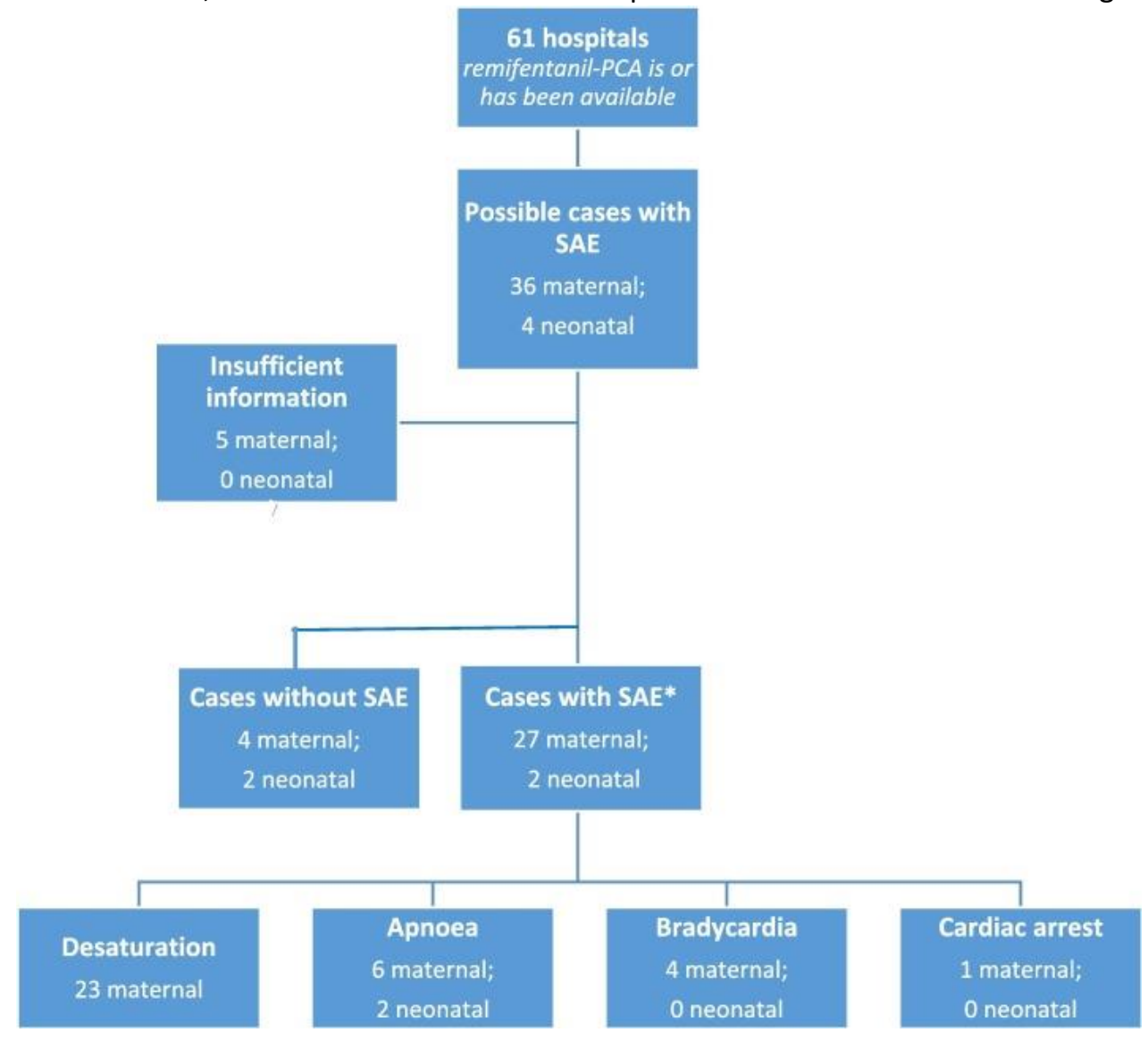


Logtenberg, S.L.M., Vink, M.L., Godfried, M.B., Beenakkers, I.C.M., Schellevis, F.G., Mol, B.W., Verhoeven, C.J. Serious adverse events attributed to remifentanil patient-controlled analgesia during labour in The Netherlands. International Journal of obstetric Anesthesia: 2019, 3, p. 22-28

Table 1. Maternal and neonatal cases with serious adverse events attributed to remifentanil patientcontrolled analgesia during labour

\begin{tabular}{|c|c|c|c|}
\hline Case & $\begin{array}{l}\text { Type of serious } \\
\text { adverse event }\end{array}$ & $\begin{array}{l}\text { Calendar } \\
\text { year(s) }\end{array}$ & Procedures followed \\
\hline $\begin{array}{l}\text { Maternal } \\
(17)\end{array}$ & Desaturation & 2007-2015 & $\begin{array}{l}\text { None or encouragement to breath; and/or stop RPCA; } \\
\text { and/or supplemental oxygen }\end{array}$ \\
\hline $\begin{array}{l}\text { Maternal } \\
(2)\end{array}$ & Desaturation; apnoea & $<2014$ & Stop RPCA; supplemental oxygen \\
\hline Maternal ${ }^{\mathrm{a}}$ & Desaturation; apnoea & 2014 & $\begin{array}{l}\text { Stop RPCA; supplemental oxygen; bag-mask } \\
\text { ventilation (three minutes); } 0.4 \text { mg naloxone } \\
\text { intravenously }\end{array}$ \\
\hline Maternal $^{b}$ & Desaturation; apnoea & 2013 & Stop RPCA; supplemental oxygen; intubation \\
\hline Maternal & Desaturation; apnoea & 2011 & $\begin{array}{l}\text { Stop RPCA; supplemental oxygen; three chest } \\
\text { compressions }\end{array}$ \\
\hline Maternal $^{\mathrm{C}}$ & $\begin{array}{l}\text { Desaturation; apnoea; } \\
\text { cardiac arrest }\end{array}$ & 2012 & $\begin{array}{l}\text { Stop RPCA; supplemental oxygen; cardiopulmonary } \\
\text { resuscitation (three minutes) }\end{array}$ \\
\hline $\begin{array}{l}\text { Maternal } \\
(3)\end{array}$ & Bradycardia & 2017 & None \\
\hline Maternal $^{d}$ & Bradycardia & 2009 & $\begin{array}{l}\text { Stop RPCA; supplemental oxygen; chest compressions } \\
\text { (a few minutes) }\end{array}$ \\
\hline Neonatal $^{\mathrm{e}}$ & Respiratory depression & 2015 & Intubation (a few hours) \\
\hline Neonatal ${ }^{f}$ & Respiratory depression & 2017 & $\begin{array}{l}\text { Five inflation breaths; positive end-expiratory } \\
\text { pressure }\end{array}$ \\
\hline
\end{tabular}

RPCA: remifentanil patient-controlled analgesia. Desaturation = oxygen saturation $\leq 85 \%$. apnoea $=$ respiratory rate $\leq 3 /$ minute. bradycardia $=$ heart rate $\leq 50$ beats/minute. cardiac arrest $=$ absence of maternal pulse. Neonatal respiratory depression = apnoea where bag-mask ventilation was applied.

administration of magnesium sulphate; oxytocin; methyldopa; nifedipine simultaneously with RPCA during labour;

${ }^{b}$ Impossible to achieve effective bag-mask ventilation;

c Error in patient-controlled analgesia pump;

${ }^{d}$ According to healthcare provider not attributed to RPCA;

e Stiff thorax diagnosed;

${ }^{f}$ Birth within three minutes of last RPCA bolus. 